\title{
Carrier and Power Assignment for Flexible Broadband GEO Satellite Communications System
}

\author{
Tedros Salih Abdu*, Eva Lagunas, Steven Kisseleff and Symeon Chatzinotas \\ Interdisciplinary Centre for Security, Reliability and Trust (SnT), \\ University of Luxembourg, Luxembourg \\ Email: \{tedros-salih.abdu, eva.lagunas, steven.kisseleff, symeon.chatzinotas\}@uni.lu \\ * Corresponding author
}

\begin{abstract}
Current multi-beam GEO satellite systems operate under a limited frequency reuse configuration and considering uniform power assignment across beams. The latter has been shown to be inefficient in matching the geographic distribution of the traffic demand. In this context, next generation of broadband GEO satellite systems will be equipped with more flexible and reconfigurable payloads, facilitating on-demand resource allocation. In this paper, we consider both carrier and power assignment to match the requested beam demands while minimizing the total transmit power and the total utilized bandwidth. A novel optimization problem is formulated and, given its non-convex structure, we divide the problem into two tractable sub-problems. First, we estimate the number of adjacent frequency carriers required for each beam to satisfy its demand and, subsequently, we optimize the power allocation based on the previously assigned carriers. We validate the proposed method with extensive numerical results, which demonstrate its efficiency with respect to benchmark strategies.
\end{abstract}

Index Terms - Flexible GEO satellite, Carrier allocation, Power allocation, Demand matching

\section{INTRODUCTION}

The application of satellite communications (SatCom) has recently evolved from providing a simple TV broadcasting to enabling broadband internet services [1]. It has an important role of providing connectivity to the maritime and aeronautical markets as well as to the remote areas with no existing connectivity to the terrestrial networks [2]. Current GEO broadband SatCom systems rely on a multibeam foot-print with limited fractional frequency reuse pattern that helps to boost the spectral efficiency while keeping the inter-beam interference under acceptable levels. In these systems, the bandwidth and power allocation is uniformly allocated across beams, rendering equal offered capacity per each beam [3]. Recent studies have shown that this uniform allocation is extremely inefficient, as the demand observed in some beams greatly exceeds the available capacity, while in others the situation is the opposite [4]. In response to the heterogeneous

This work has been partially supported by the Luxembourg National Research Fund (FNR) under the project FlexSAT (C19/IS/13696663), the project ROSETTA (11632107), and the ANR-FNR project SIERRA.

978-1-7281-4490-0/20/\$31.00 @ 2020 IEEE traffic demand patterns, next generation of SatCom platforms incorporate highly flexible and automatically reactive payloads, able to reconfigure frequency and power allocation dynamically at anytime during the satellite mission [5], [6].

Flexible satellite resource allocation strategies for the supply-demand matching problem has recently attracted attention from the research community. Beam bandwidth assignment based on beam traffic demand has been addressed in [7], while the power optimization alternative has been considered in [8], [9]. However, inter-beam interference has not been considered in these works. Dynamic power allocation was studied in [10]-[13] considering the conventional frequency reuse pattern, where inter-beam interference is minimized at the cost of limited spectral efficiency.

A joint power and frequency allocation is introduced in [14], [15] with the assumption on orthogonal carrier assignment within a beam cluster and a binary power allocation. The benefits of joint power and frequency allocation are explored in [15], [16] but considering an additional degree of flexibility over time domain provided by Beam-Hoping (BH), thus deactivating beams that are close together generating high levels of co-channel interference. Focusing on the frequency flexibility and the power optimization, the recent works in [17], [18] propose computationally expensive algorithms targeting unmet system capacity (or satisfaction-gap) minimization.

In this paper, we consider both carrier and power assignment to match the requested beam demands while minimizing the total transmit power and the total utilized bandwidth. The objective behind this design approach is to determine the minimum satellite resources required to satisfy the demand. Note that the final assignment will intentionally push for high frequency reuse as far as the generated interference is not preventing the offered capacity to achieve the requested demand. In addition, minimizing the satellite power consumption is a fundamental aspect, particularly for the upcoming digitallycontrolled payloads with on-board active antenna [19], [20].

To this end, we formulate a novel optimization problem, which is found to be NP-hard and thus difficult to solve. Given its non-convex structure, we divide the problem into two 
more tractable sub-problems. We first estimate the number of adjacent frequency carriers required for each beam to satisfy its demand and, subsequently, we optimize the power allocation based on the previously assigned carriers. The proposed strategy is compared with benchmark allocation techniques via numerical experiments and the results evidence its efficiency in adapting to different demand distributions.

The remainder of the paper is organized as follows. Section II introduces the system model. The problem formulation and the proposed solution are presented in Section III and Section IV, respectively. Supporting numerical results are provided in Section V, and Section VI states the conclusion.

Notation: Boldface of upper case and lower case letters refers to matrices and vectors, respectively. The symbol $\odot$ represents the Hadamard element-wise product. The transpose of a vector and conjugate transpose of a vector represented by $[.]^{T}$ and $[.]^{H}$, respectively. Lastly, $\mathbb{E}[$.$] and \operatorname{diag}($.$) represents$ the expected value and a diagonal matrix, respectively.

\section{System MODEL}

Consider a downlink multibeam GEO satellite system with $N$ beams and $K$ carriers $(\mathcal{K}=\{1,2, \ldots, K\})$ as shown in the Fig. 1. Each carrier has bandwidth $B_{s c}$ such that the total available system bandwidth is equal to $B_{t o t}=K \cdot B_{s c}$. In our analysis, we focus on a single super-user per beam, which is assumed to aggregate the overall beam demand. Since the offered capacity is dependent on the user location, different realizations will be considered in the simulation results section by changing the super-user location. Henceforth, we will use the term beam and user interchangeably.

The channel vector from the satellite $i$ th beam over the $k$ th carrier is denoted by $\mathbf{h}_{i, k}=\left[h_{i, k}[1], h_{i, k}[2], \ldots, h_{i, k}[N]\right]^{T}$. We denote $\mathbf{x}_{k}=\left[x_{k}[1], x_{k}[2], \ldots, x_{k}[N]\right]^{T}$ the assignment vector for carrier $k$, where $x_{k}[i] \in\{0,1\}$ with $x_{k}[i]=1$ indicating that carrier $k$ is assigned to the $i$ th beam. Similarly, the transmit power allocation vector for $k$ th carrier is denoted as $\mathbf{p}_{k}=\left[p_{k}[1], p_{k}[2], \ldots, p_{k}[N]\right]^{T}$, where $p_{k}[i]$ is the transmit power of $k$ th carrier in the $i$ th beam. The information symbols to be transmitted in the $k$ th carrier are arranged in $\mathbf{s}_{k}=\left[s_{k}[1], s_{k}[2], \ldots, s_{k}[N]\right]^{T}$. Hence, the received signal at $i$ th beam from the $k$ th carrier can be expressed as

$$
y_{k}[i]=\mathbf{h}_{i, k}^{T} \sqrt{\mathbf{P}}\left[\mathbf{x}_{k} \odot \mathbf{s}_{k}\right]+n_{k}[i]
$$

where $\mathbf{P}=\operatorname{diag}\left(\mathbf{p}_{k}\right)$ and $n_{k}[i]$ denotes the additive white Gaussian noise at beam $i$ for carrier $k$. The average power of the transmitted symbols is assumed normalized to one, i.e. $\mathbb{E}\left[\mathbf{s}_{k} \mathbf{s}_{k}^{H}\right]=\mathbf{I}_{N}$. By reformulating (1), we obtain

$$
\begin{aligned}
y_{k}[i] & =\underbrace{\sqrt{p_{k}[i]} h_{i, k}[i] x_{k}[i] s_{k}[i]}_{\text {Desired Signal }} \\
& +\underbrace{\sum_{j=1, j \neq i}^{N} \sqrt{p_{k}[j]} h_{i, k}[j] x_{k}[j] s_{k}[j]}_{\text {Interference Signal }}+n_{k}[i] .
\end{aligned}
$$

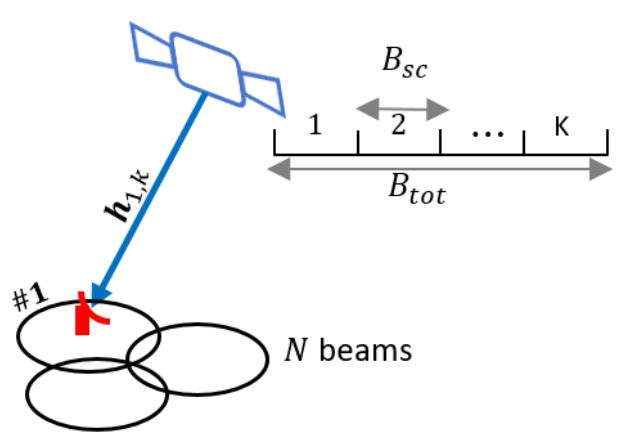

Fig. 1. Multibeam satellite communication over given carriers.

The channel coefficients $h_{i, k}[j]$ are defined as

$$
h_{i, k}[j]=\frac{\sqrt{G_{R} G_{i}[j]}}{4 \pi \frac{d_{i}}{\lambda}},
$$

where $G_{R}$ is the user terminal antenna gain, $G_{i}[j]$ denotes the gain from the $j$ th satellite antenna towards the $i$ th user and $d_{i}$ is the slant range between the satellite and the $i$ th user.

The signal-to-interference-plus-noise ratio (SINR) of the $i$ th beam over $k$ th carrier is given by

$$
\gamma_{i, k}\left(\mathbf{p}_{k}, \mathbf{x}_{k}\right)=\frac{g_{i, k}[i] p_{k}[i] x_{k}[i]}{\sum_{j=1, j \neq i}^{N} g_{i, k}[j] p_{k}[j] x_{k}[j]+\sigma^{2}},
$$

where $g_{i, k}[j]$ denotes the channel power gain $g_{i, k}[j]=$ $\left|h_{i, k}[j]\right|^{2}$ and $\sigma^{2}$ is the noise power of $n_{k}[i]$. Hence, the offered capacity at beam $i$ assuming carrier $k$ is given by

$$
C_{i, k}=B_{s c} \log _{2}\left(1+\gamma_{i, k}\left(\mathbf{p}_{k}, \mathbf{x}_{k}\right)\right),
$$

and the overall offered capacity at beam $i$ considering the aggregation of all available carriers of the system is

$$
C_{i}=\sum_{k=1}^{K} C_{i, k}
$$

\section{PROBLEM Formulation}

Our objective is to satisfy the beam traffic demands making use of the minimum transmit power and the minimum number of carriers. Regarding the power constraints, we constraint the total radiated power to be below $P_{\text {total }}$ and the power per beam to be below $P_{\max }$. In other words, the power and carrier assignment $\mathbf{x}_{k}, \mathbf{p}_{k}, k=1, \ldots, K$, shall be given by the solution to the following optimization problem,

$$
\underset{\mathbf{x}_{k}, \mathbf{p}_{k}}{\operatorname{minimize}} \sum_{i=1}^{N} \sum_{k=1}^{K} x_{k}[i]+\sum_{i=1}^{N} \sum_{k=1}^{K} p_{k}[i]
$$

subject to:

$$
\begin{aligned}
& R 1: \sum_{k=1}^{K} C_{i, k} \geq D_{i}, \forall_{i} \\
& R 2: \sum_{i=1}^{N} \sum_{k=1}^{K} p_{k}[i] \leq P_{\text {total }}
\end{aligned}
$$




$$
\begin{aligned}
& R 3: \sum_{k=1}^{K} p_{k}[i] \leq P_{\text {max }}, \forall_{i}, \\
& R 4: x_{k}[i] \in\{0,1\}, \forall_{i}, \forall_{k}, \\
& R 5: p_{k}[i] \leq x_{k}[i] P_{\max }, \forall_{i}, \forall_{k}, \\
& R 6: p_{k}[i] \geq 0, \forall_{i}, \forall_{k},
\end{aligned}
$$

The problem in (7) contains 6 constraints, which are explained in detail in the following:

- $R 1$ guarantees that the total offered capacity in each beam satisfies the respective demand $D_{i}$;

- $R 2$ refers to the overall satellite transmit power, which should not exceed the total available power $P_{\text {total }}$;

- $R 3$ is the per-beam power constraint, where each beam should not exceed the maximum power $P_{\max }$ per beam;

- $R 4$ indicates a binary carrier assignment $x_{k}[i]$;

- $R 5$ guarantees that power is allocated only if the carrier is assigned, i.e. $x_{k}[i]=1$. Note that $R 5$ allow as to re-write $\gamma_{i, k}\left(\mathbf{p}_{k}, \mathbf{x}_{k}\right)$ as $\gamma_{i, k}\left(\mathbf{p}_{k}\right)=\frac{g_{i, k}[i] p_{k}[i]}{\sum_{j=1, j \neq i}^{N} g_{i, k}[j] p_{k}[j]+\sigma^{2}}$.

- $R 6$ ensures that the assigned power is non-negative.

The optimization problem (7) is clearly non-convex because of $R 1$ and $R 4$. In particular, the non-convexity of $R 1$ arises from the non-linearity of the SINR $\gamma_{i, k}\left(\mathbf{p}_{k}\right)$. The non-convexity of $R 4$ is due to the binary carrier indicator $\left(x_{k}[i]\right)$, which makes (7) a mixed-integer program. Due to the non-convexity of (7), it is not possible to obtain the optimal solution via well-known tools of convex optimization, cf. [21]. Hence, in the following, we propose a high performance sub-optimal solution with reasonable complexity.

\section{Proposed Carrier And Power Allocation}

Due to the complexity of the original optimization problem (7), in this section we propose a sub-optimal approach by splitting the original problem into two tractable sub-problems. The first sub-problem addresses the design of the carrier assignment variables $\mathbf{x}_{k}$, while the second sub-problem focuses on the overall consumed power minimization by designing the variables $\mathbf{p}_{k}$, based on the predefined carrier allocation obtained in the first step.

\section{A. Step 1: Carrier Assignment}

In this section, we first obtain the number of carriers $K_{i}\left(1 \leq K_{i} \leq K\right)$ required for the $i$ th beam to match the requested demand. Then we propose a simple algorithm to map the number of active carriers $K_{i}$ into the respective variables $\mathbf{x}_{k}$, while minimizing the overall number of carriers.

Due to the assumed flat fading channel, we can exploit the similarity of the channel at different carriers of the same $i$ th beam by expressing $\mathbf{h}_{i, k} \approx \hat{\mathbf{h}}_{i}$. Note that the atmospheric impairments usually affect the channel across a large signal spectrum in a similar way, such that the losses within the considered frequency band (e.g. $B_{\text {tot }}=500 \mathrm{MHz}$ ) can be viewed as sufficiently uniform. Hence, it is likely that the SINR values in all carriers $k=1, \ldots, K$ of beam $i$ are similar, such that we can approximate $\gamma_{i, k}\left(\mathbf{p}_{k}\right)$ by $\hat{\gamma}_{i}(\mathbf{p})$, and (6) converts into

$$
\begin{aligned}
C_{i}=\sum_{k=1}^{K} C_{i, k} & =\sum_{k=1}^{K} B_{s c} \log _{2}\left(1+\gamma_{i, k}\left(\mathbf{p}_{k}\right)\right) \\
& \approx K_{i} B_{s c} \log _{2}\left(1+\hat{\gamma}_{i}(\mathbf{p})\right) \\
\text { with } \hat{\gamma}_{i}(\mathbf{p}) & =\frac{g_{i}[i] p[i]}{\sum_{j=1, j \neq i}^{N} g_{i}[j] p[j]+\sigma^{2}}
\end{aligned}
$$

with $\mathbf{p}=[p[1], p[2], \ldots, p[N]]^{T}$, and $g_{i}[j]=\left|\hat{h}_{i}[j]\right|^{2}$. Note that $p[i]$ denotes equal transmit power per carrier in the $i$ th beam. Hence, for a feasible interpretation of the results, the values of $p_{k}[i]$ must not exceed $x_{i, k} p[i]$.

Using (8), we formulate a similar problem as in (7) but replacing the sum of all carrier assignment variables $\mathbf{x}_{k}[i]$ by the number of carriers $K_{i}, i=1, \ldots, N$. The resulting problem is formulated as follows:

$$
\underset{K_{i}, \mathbf{p}}{\operatorname{minimize}} \sum_{i=1}^{N} K_{i}+\sum_{i=1}^{N} K_{i} p[i]
$$

subject to:

$$
\begin{aligned}
& V 1: K_{i} B_{s c} \log _{2}\left(1+\hat{\gamma}_{i}(\mathbf{p})\right) \geq D_{i}, \forall_{i}, \\
& V 2: \sum_{i=1}^{N} K_{i} p[i] \leq P_{\text {total }} \\
& V 3: K_{i} p[i] \leq P_{\text {max }}, \forall_{i}, \\
& V 4: K_{i} \in \mathcal{K}, \forall_{i} \\
& V 5: p[i] \geq 0, \forall_{i}
\end{aligned}
$$

Problem (10) is still non-convex because of

- the interference term in $\hat{\gamma}_{i}(\mathbf{p})$,

- the product of $K_{i} p[i]$ in $V 2$,

- the integer value of $K_{i}$ in $V 4$.

Hence, we propose a method to convexify (10). We start by approximating the integer constraint $V 4$ as a continuous function in the range between 1 and $K$. Next, we replace $p[i]$ and $K_{i}$ with the exponential functions $\exp (q[i])$ and $\exp \left(Z_{i}\right)$, respectively. Constraint $V 1$ can be then reformulated as two constraints:

$$
\begin{aligned}
& V 1.1: D_{i} \exp \left(-Z_{i}\right)-B_{s c} \log _{2}(1+\exp (\alpha[i])) \leq 0, \forall_{i} \\
& V 1.2: \exp (\alpha[i]) \leq \frac{g_{i}[i] \exp (q[i])}{\sum_{j=1, j \neq i}^{N} g_{i}[j] \exp (q[j])+\sigma^{2}}, \forall_{i}
\end{aligned}
$$

where $\exp (\alpha[i])$ is the upper bound of $\hat{\gamma}_{i}(\mathbf{p})$. By applying logarithm on $V 1.2$ constraint, we obtain

$$
\begin{aligned}
V 1.3: & \alpha[i]-q[i]-\log \left(g_{i}[i]\right) \\
& +\log \left(\sum_{j=1, j \neq i}^{N} g_{i}[j] \exp (q[j])+\sigma^{2}\right) \leq 0, \forall_{i} .
\end{aligned}
$$

Since the log-sum-exp type of functions are convex (cf. [21]), (12) is convex. Unfortunately, constraint $V 1.1$ remains nonconvex. However, we observe that $V 1.1$ contains a difference 
of convex functions. Thus, a typical method (cf. [22]) for solving a Difference-of-Convex-Functions (DC) Program can be applied in order to convexify $V 1.1$. First, we reformulate

$$
V 1.1: J_{1}\left(Z_{i}\right)-J_{2}(\alpha[i]) \leq 0, \forall_{i},
$$

where both $J_{1}\left(Z_{i}\right)=D_{i} \exp \left(-Z_{i}\right)$ and $J_{2}(\alpha[i])=$ $B_{s c} \log _{2}(1+\exp (\alpha[i]))$ are convex functions. The DC programming can be tackled using Successive Convex Approximation (SCA) algorithm by approximating the concave part [23]. The first order approximation of $J_{2}(\alpha[i])$ in (13) is given by

$$
\widetilde{J}_{2}(\alpha[i])=J_{2}\left(\alpha[i]^{(l)}\right)+\nabla J_{2}\left(\alpha[i]^{(l)}\right)\left(\alpha[i]-\alpha[i]^{(l)}\right),
$$

where $\alpha[i]^{(l)}$ is the value of $\alpha[i]$ used in the $l$ th iteration of SCA and

$$
\begin{aligned}
J_{2}\left(\alpha[i]^{(l)}\right) & =B_{s c} \log _{2}\left(1+\exp \left(\alpha[i]^{(l)}\right)\right), \\
\nabla J_{2}\left(\alpha[i]^{(l)}\right) & =B_{s c} \frac{1}{\log (2)}\left(\frac{\exp \left(\alpha[i]^{(l)}\right)}{1+\exp \left(\alpha[i]^{(l)}\right)}\right) .
\end{aligned}
$$

The overall optimization problem (10) is reformulated as

$$
\underset{Z_{i}, \mathbf{q}}{\operatorname{minimize}} \sum_{i=1}^{N} \exp \left(Z_{i}\right)+\sum_{i=1}^{N} \exp \left(q[i]+Z_{i}\right)
$$

subject to:

$$
\begin{aligned}
& V 1.1^{\prime}: J_{1}\left(Z_{i}\right)-\widetilde{J}_{2}(\alpha[i]) \leq 0, \forall_{i}, \\
& V 1.3, \\
& V 2: \sum_{i=1}^{N} \exp \left(q[i]+Z_{i}\right) \leq P_{\text {total }}, \\
& V 3: \exp \left(q[i]+Z_{i}\right) \leq P_{\text {max }}, \forall_{i}, \\
& V 4^{\prime}: 1 \leq \exp \left(Z_{i}\right) \leq K, \forall_{i}, \\
& V 5: \exp (q[i]) \geq 0, \forall_{i},
\end{aligned}
$$

Both $V 4^{\prime}$ and $V 1.1^{\prime}$ are the continuous value approximation of $V 4$ and the first order approximation of $V 1.1$, respectively. This problem is now convex and can be solved optimally. The iterative process to obtain the number of carriers required for each beam to match the traffic demand is detailed in Alg. 1.

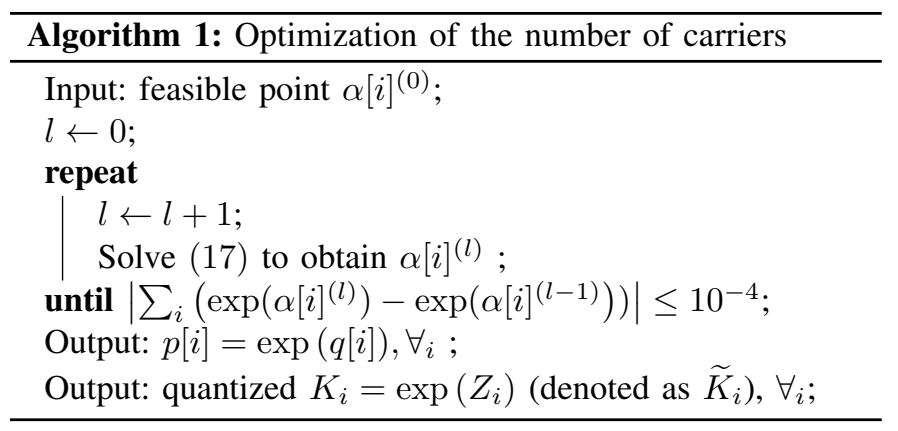

We start with a feasible point of $\alpha[i]^{(0)}=\log \left(\gamma_{i}(\mathbf{p})\right)$. By solving (17) we obtain a new point $\alpha[i]^{(l+1)}$. The algorithm terminates when the absolute sum of the difference between $\exp \left(\alpha[i]^{(l)}\right)$ and $\exp \left(\alpha[i]^{(l-1)}\right)$ becomes very small $\left(10^{-4}\right.$ in this work), which indicates the convergence of the algorithm.

Note that the solution $K_{i}=\exp \left(Z_{i}\right)$ of (17) in each iteration may have decimal values. Since $K_{i}$ should be an integer number of carriers, in the last step of Alg. 1, we quantize $K_{i}$ via $\widetilde{K}_{i}=\left\lceil K_{i}-0.1\right\rceil$, where $\lceil\cdot\rceil$ is the ceiling operator, which provides the smallest integer larger than its argument. The quantization of $K_{i}$ needs to be carefully chosen in order to maximize the system performance. In our investigation, we observed that the best results are obtained with the quantization strategy mentioned above.

In the following, we employ the optimized number of carriers $\widetilde{K}_{i}$ in order to obtain the carrier assignments $x_{i, k}$. All values of $x_{i, k}$ are initially set to zero. We fill the carrier assignments into vector $\mathbf{x}_{k}$ by setting $x_{i, k}=1, k=1,2, \ldots, \widetilde{K}_{i}$. Note that we intentionally packed contiguous carriers for each of the beam, since this is known to ease the hardware implementation by assuming a single wider carrier instead of multiple separated narrow carriers [24]. The described carrier assignment procedure is also depicted in Alg. 2.

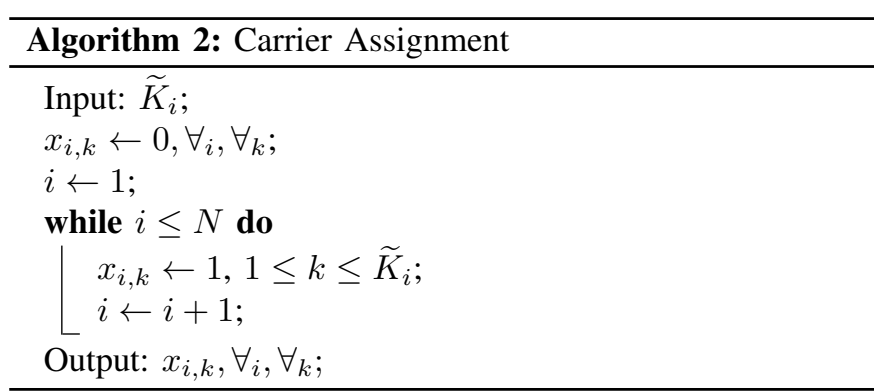

\section{B. Step 2: Power Optimization for Active Carriers}

After having optimized the carrier assignment $\left(\mathbf{x}_{k}\right)$ and obtained an upper-bound on the power per carrier in each beam $(p[i])$, in this section we focus on the optimization of the transmit power per beam. The optimization problem is formulated as follows

$$
\underset{\mathbf{p}_{k}}{\operatorname{minimize}} \sum_{i=1}^{N} \sum_{k=1}^{K} p_{k}[i]
$$

subject to:

$$
\begin{aligned}
& R 1: D_{i}-\sum_{k=1}^{K} B_{s c} \log _{2}\left(1+\gamma_{i, k}\left(\mathbf{p}_{k}\right)\right) \leq 0, \forall_{i}, \\
& R 2: \sum_{i=1}^{N} \sum_{k=1}^{K} p_{k}[i] \leq P_{\text {total }}, \\
& R 3: \sum_{k=1}^{K} p_{k}[i] \leq P_{\max }, \forall_{i}, \\
& R 6: p_{k}[i] \geq 0, \forall_{i}, \forall_{k}, \\
& R 7: p_{k}[i] \leq x_{k}[i] p[i], \forall_{i}, \forall_{k},
\end{aligned}
$$

The optimization problem (18) is non-convex because of constraint $R 1$. However, similar to (13), this constraint can 
be reformulated in terms of DC programming. Furthermore, we express $R 1$ as an exponential function $\exp \left(\beta_{k}[i]\right)$, which is upper bounded by $\gamma_{i, k}\left(\mathbf{p}_{k}\right)$ :

$$
\begin{aligned}
& R 1.1: D_{i}-\sum_{k=1}^{K} B_{s c} \log _{2}\left(1+\exp \left(\beta_{k}[i]\right)\right) \leq 0, \forall_{i} \\
& R 1.2: \exp \left(\beta_{k}[i]\right) \leq \gamma_{i, k}\left(\mathbf{p}_{k}\right), \forall_{i}, \forall_{k}
\end{aligned}
$$

Both $R 1.1$ and $R 1.2$ in (19) are non-convex. In order to convexify $R 1.2$ we replace the power $p_{k}[i]$ by $\exp \left(q_{k}[i]\right)$. Hence, it is possible to apply logarithm on both sides of the equation and obtain

$$
\begin{aligned}
& R 1.3: \beta_{k}[i]-q_{k}[i]-\log \left(g_{i, k}[i]\right) \\
& \quad+\log \left(\sum_{j=1, j \neq i}^{N} g_{i, k}[j] \exp \left(q_{k}[j]\right)+\sigma^{2}\right) \leq 0, \forall_{i}, \forall_{k},
\end{aligned}
$$

In the following, we denote $\boldsymbol{\beta}=\left[\beta_{1}[i], \beta_{2}[i], \ldots, \beta_{K}[i]\right]^{T}$. The constraint $R 1.1$ contains a difference of convex functions. Thus, by approximating its concave part we obtain

$$
R 1.4: D_{i}-\widetilde{Q}(\boldsymbol{\beta}) \leq 0, \forall_{i}
$$

where $\widetilde{Q}(\boldsymbol{\beta})$ is the first-order approximation of $Q(\boldsymbol{\beta})$ :

$$
\begin{aligned}
\widetilde{Q}(\boldsymbol{\beta}) & =Q\left(\boldsymbol{\beta}^{(l)}\right)+\nabla Q\left(\boldsymbol{\beta}^{(l)}\right)\left(\boldsymbol{\beta}-\boldsymbol{\beta}^{(l)}\right), \\
Q\left(\boldsymbol{\beta}^{(l)}\right) & =\sum_{m=1}^{K} B_{s c} \log _{2}\left(1+\exp \left(\beta_{m}[i]^{(l)}\right)\right), \\
\nabla Q\left(\boldsymbol{\beta}^{(l)}\right) & =\frac{1}{\log (2)}\left[\begin{array}{c}
\frac{\exp \left(\beta_{1}[i]^{(l)}\right)}{1+\exp \left(\beta_{1}[i]^{(l)}\right)} \\
\vdots \\
\frac{\exp \left(\beta_{K}[i]^{(l)}\right)}{1+\exp \left(\beta_{K}[i]^{(l)}\right)}
\end{array}\right]^{T} .
\end{aligned}
$$

Since the approximation of $Q(\boldsymbol{\beta})$ is repeated iteratively by the SCA algorithm, we denote $\boldsymbol{\beta}^{(l)}$ the value of $\boldsymbol{\beta}$ in $l$ th iteration. After these modifications, the second sub-problem can be formulated as:

$$
\underset{\mathbf{p}_{k}}{\operatorname{minimize}} \sum_{i=1}^{N} \sum_{k=1}^{K} p_{k}[i]
$$

subject to: $R 1.3, R 1.4, R 2, R 3, R 6, R 7$

This problem is convex and we obtain a solution using an iterative based on SCA, which is shown in Alg. 3. Similar to Alg. 1, we initialize the variables based on a feasible point corresponding to $\exp \left(\beta_{k}[i]^{(0)}\right)=\gamma_{i, k}\left(\mathbf{p}_{\boldsymbol{k}}\right)$ and set the iteration counter to $l=0$. Then, $(25)$ is solved, which leads to a new value $\beta_{k}[i]^{(l+1)}$. The algorithm terminates when the absolute sum of the difference between $\exp \left(\beta_{k}[i]^{(l)}\right)$ and $\exp \left(\beta_{k}[i]^{(l-1)}\right)$ is below $10^{-4}$, which indicates the convergence of this algorithm.

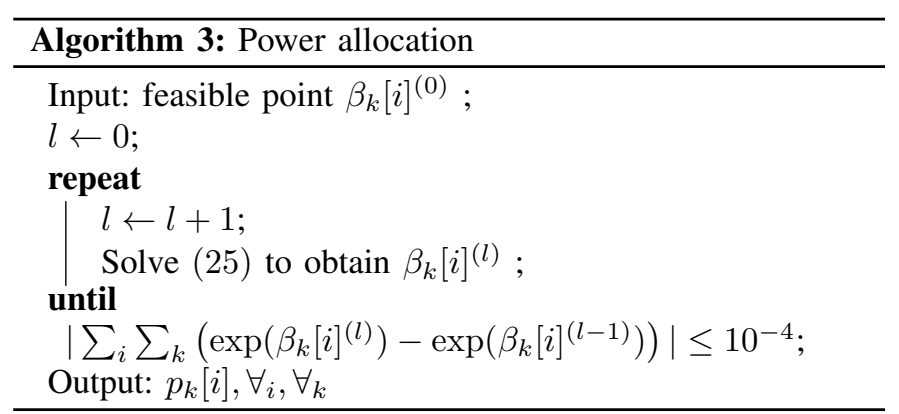

\section{Simulation Results}

In this section, we evaluate the performance of the proposed carrier and power allocation (CPA) method through simulations. Table I summarizes the key simulation parameters. The results shown in this section have been obtained from $M=176$ realizations. In each realization, the locations of the super-user terminals have been selected randomly from a uniform distribution within the considered beam coverage. An example of super-user distribution is depicted in Fig. 2.

TABLE I

SySTEM PARAMETERS

\begin{tabular}{|c|c|}
\hline Parameter & Value \\
\hline Satellite Orbit & $13^{\circ} \mathrm{E}$ \\
\hline Number of beams $(N)$ & 7 \\
\hline Number of carrier $(K)$ & 4 \\
\hline System Bandwidth $\left(B_{t o t}\right)$ & $500 \mathrm{MHZ}$ \\
\hline Sub-carrier bandwidth $\left(B_{S C}\right)$ & $125 \mathrm{MHz}$ \\
\hline Noise power $\left(\sigma^{2}\right)$ & $-123 \mathrm{dBW}$ \\
\hline Max. beam gain $\left(G_{i}[j]\right)$ & $51.8 \mathrm{dBi}$ \\
\hline User antenna gain $\left(G_{R}\right)$ & $39.8 \mathrm{dBi}$ \\
\hline Total Power of the system $\left(P_{\text {total }}\right)$ & $500 \mathrm{~W}$ \\
\hline Maximum power for each beam $\left(P_{\max }\right)$ & $100 \mathrm{~W}$ \\
\hline
\end{tabular}

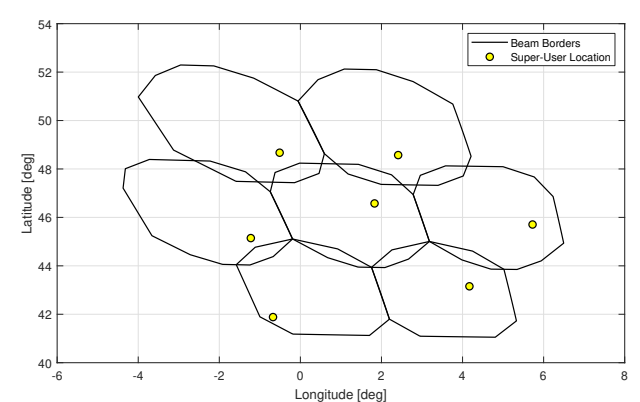

Fig. 2. $N=7$ beam scenario with an example of super-user distribution

For comparison, we consider the following benchmark schemes:

1) Scheme 1: Single polarization four color reuse scheme with uniform power allocation across the beams $p_{s 1}[i]=$ $P_{\text {total }} / N$.

2) Scheme 2: Single polarization four color reuse scheme with demand based non-uniform power allocation across the beams:

$$
p_{s 2}[i]=\frac{\left(2^{\frac{D_{i}}{B_{s c}}}-1\right) \sigma^{2}}{g_{i}[i]} .
$$


Note: proper scaling of $p_{s 2}[i]$ is required to satisfy $(R 2)$ and (R3).

3) Scheme 3: Single polarization four color reuse factor with demand based uniform power allocation across the beams $p_{s 3}[i]=\max _{i}\left(p_{s 2}[i]\right)$. Note: proper scaling of $p_{s 3}[i]$ is required to satisfy $(R 2)$ and $(R 3)$.

The performance is evaluated with respect to:

\section{1) Average satisfaction index (ASI)}

$$
A S I=\frac{1}{N M} \sum_{m=1}^{M} \sum_{n=1}^{N} S I_{i}[m]
$$

where $M$ is the number of channel realizations and $S I_{i}[m]$ is the satisfaction index of $i$ th beam in $m$ th realization given by $S I_{i}[m]=\min \left(\frac{\sum_{k=1}^{K} C_{i, k}[m]}{D_{i}}, 1\right)$.

\section{2) Average used power (AUP)}

- For the proposed method:

$$
A U P=\frac{1}{M} \sum_{m=1}^{M} \sum_{i=1}^{N} \sum_{k=1}^{K} p_{k}[i] .
$$

- For the three benchmark schemes (single carrier per beam) with $p_{s}[i] \in\left\{p_{s 1}[i], p_{s 2}[i], p_{s 3}[i]\right\}$ :

$$
A U P=\frac{1}{M} \sum_{m=1}^{M} \sum_{i=1}^{N} p_{s}[i]
$$

\section{A. Uniform per-Beam Demand}

We assume that all beams have the same demand $D_{i}=D$, $\forall i$, and we vary the value of $D$ between 100 and 1000 Mbps. Fig. 3 shows the ASI of users for CPA and benchmark schemes. We observe, that the performance of all schemes degrades with increasing demand. This degradation results in general from the fact that for some channel realizations it is physically impossible to satisfy the demand even by increasing the transmit power due to the interference between the beams. Correspondingly, with increasing demand, we obtain more and more realizations, for which the formulated optimization problem is infeasible. In such cases, in order to obtain a solution, we reduce the imposed demand until the optimization problem becomes feasible. Accordingly, the offered capacity relative to the true demand, i.e. ASI, decreases, which can be observed in Fig. 3 starting from 700 Mbps. Nevertheless, the proposed CPA method outperforms all benchmark schemes, such that we obtain an improvement in demand matching starting from $600 \mathrm{Mbps}$.

Interestingly, the proposed method not only shows a better demand matching compared to benchmark schemes, but also requires less overall transmit power. Fig. 4 shows the results for the AUP. All three benchmark schemes converge to the same AUP, which is related to the total power $P_{\text {total }}$. In contrast, the proposed method converges to a different AUP, which is $\approx 7 \mathrm{~dB}$ less than for the benchmark schemes. Correspondingly, we observe a performance gain in terms of AUP, especially for high demand. For low demand, i.e. below $200 \mathrm{Mbps}$, the benchmark scheme 2 seems to require less

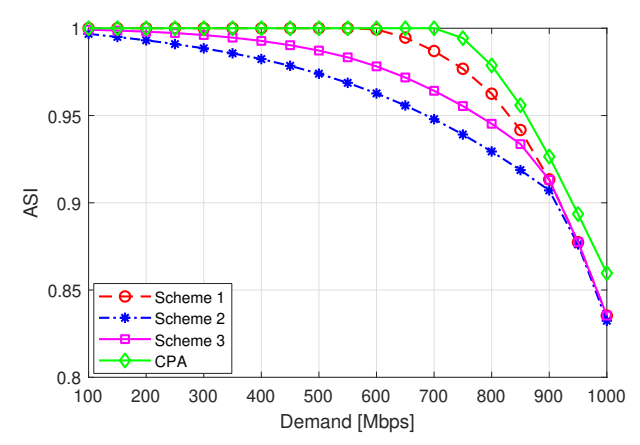

Fig. 3. ASI comparison of CPA and benchmark schemes for different demand of users

transmit power than the proposed method (by at most $1 \mathrm{~dB}$ ). However, this is compensated by a better satisfaction index with the proposed method.

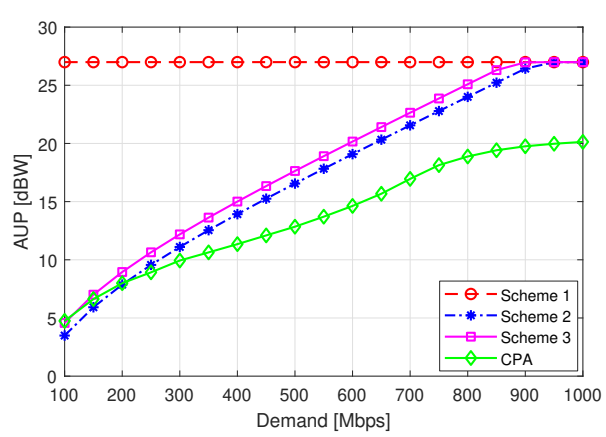

Fig. 4. AUP comparison of CPA and benchmark schemes for different demand of users

Next, we would like to investigate the number of carriers allocated per-beam depending on the demand $D$. The average number of carriers over $M$ realizations is shown in Table II. In particular, Table II provides the percentage of beams that are allocated either 1,2,3 or all 4 carriers. We observe the dependency of the number of allocated carriers on the traffic demand. When $D=100 \mathrm{Mbps}$, only $K_{i}=1$ carrier is used in $99.43 \%$ of cases, whereas $K_{i}=2$ carriers are utilized in $0.57 \%$ of cases. As the demand increases, the algorithm assigns more and more carriers to satisfy the requested demand until it reaches the full-frequency reuse, where all carriers are used in all beams.

\section{B. Non-Uniform per-Beam Demand}

In this section, we compare the proposed scheme with the benchmark schemes for single channel realization when the per-beam demand is non-uniform. Fig. 5 shows the per-beam demand matching results, where it can be observed that the proposed method satisfies the user demand while benchmark schemes fail. In particular, the offered capacity of scheme 1 is less than the demand in beams 2 and 3 . With scheme 2 , the capacity is less than the demand in beams $2,3,5$ and 6 . Scheme 3 fails in beams 2, 3 and 6 . 
TABLE II

NUMBER OF CARRIERS VERSUS DEMAND (IN PERCENT)

\begin{tabular}{|c|c|c|c|c|}
\hline \multirow{2}{*}{ Mbps } & \multicolumn{4}{|c|}{ Carriers $\left(K_{i}\right)$} \\
\cline { 2 - 5 } & 1 & 2 & 3 & 4 \\
\hline 100 & 99.43 & 0.57 & 0 & 0 \\
\hline 150 & 11.93 & 88.07 & 0 & 0 \\
\hline 200 & 0 & 90.9 & 9.1 & 0 \\
\hline 250 & 0 & 32.95 & 66.48 & 0.57 \\
\hline 300 & 0 & 9.66 & 78.4 & 11.94 \\
\hline 350 & 0 & 0.57 & 45.45 & 53.98 \\
\hline 400 & 0 & 0 & 17.6 & 82.4 \\
\hline 450 & 0 & 0 & 7.95 & 92.05 \\
\hline 500 & 0 & 0 & 0.57 & 99.43 \\
\hline 550 & 0 & 0 & 0 & 100 \\
\hline 600 & 0 & 0 & 0 & 100 \\
\hline 650 & 0 & 0 & 0 & 100 \\
\hline 700 & 0 & 0 & 0 & 100 \\
\hline
\end{tabular}

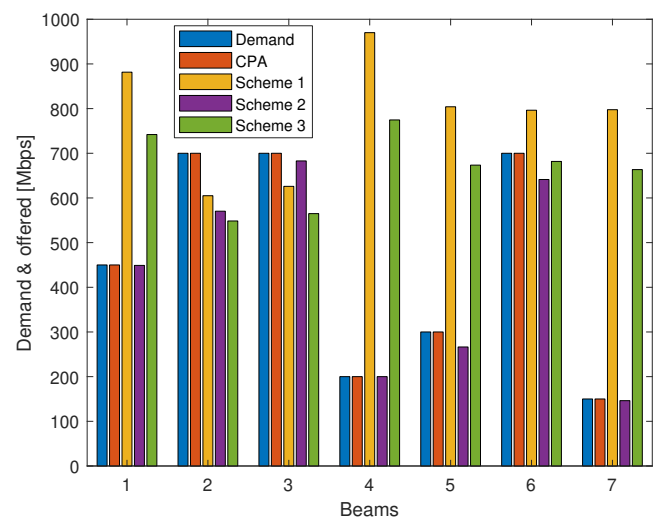

Fig. 5. Offered capacity comparison of CPA and benchmark schemes to match demand per beam

\section{CONCLUSIONS}

In this paper, we proposed a novel method of carrier and power assignment for flexible broadband GEO SatCom systems in order to match the requested demand while using the minimum total power and the minimum number of carriers of the system. Since the respective optimization problem is non-convex in general, we split the problem in multiple subproblems and solved them iteratively. The proposed scheme shows a better performance compared to the benchmark schemes in terms of both average per-beam demand matching and average consumed power.

\section{ACKNOWLEDGMENT}

We would like to express our gratitude to SES for a great support and specifically to Dr. Joel Grotz for valuable discussions and suggestions.

\section{REFERENCES}

[1] S. K. Sharma, S. Chatzinotas, and P.-D. Arapoglou, Satellite Communications in the 5G Era. IET Digital Library, 2018.

[2] O. Kodheli et al., "Satellite Communications in the New Space Era: A Survey and Future Challenges," Feb. 2020, arXiv:2002.08811.

[3] W. Wu, "Satellite Communications," Proceedings of the IEEE, vol. 85 no. 6, pp. 998-1010, Jun. 1997.

[4] ESA FlexPreDem Project: Demostrator of Precoding Techniques for Flexible Broadband Systems. [Online]. Available: https://artes.esa.int/projects/flexpredem
[5] "EUTELSAT 172b Satellite: On the Road to Kourou, March 2017," https://news.eutelsat.com/pressreleases/eutelsat-172b-satellite-on-theroad-to-kourou-1857558, accessed: 2019-12.

[6] "SES and Thales Unveil Next Generation Capabilities On-Board SES-17, April 2017," https://www.ses.com/press-release/ses-and-thalesunveil-next-generation-capabilities-onboard-ses-17, accessed: 2020-02.

[7] U. Park, H. W. Kim, D. S. Oh, and B. J. Ku, "Flexible Bandwidth Allocation Scheme Based on Traffic Demands and Channel Conditions for Multi-Beam Satellite Systems," in Proc. of IEEE Vehicular Technology Conference (VTC Fall), Sep. 2012, pp. 1-5.

[8] J. P. Choi and V. W. S. Chan, "Optimum power and beam allocation based on traffic demands and channel conditions over satellite downlinks," IEEE Transactions on Wireless Communications, vol. 4, no. 6 , pp. 2983-2993, Nov 2005.

[9] T. Qi and Y. Wang, "Energy-efficient power allocation over multibeam satellite downlinks with imperfect CSI," in Proc. of International Conference on Wireless Communications Signal Processing (WCSP), Oct 2015, pp. 1-5.

[10] N. K. Srivastava and A. K. Chaturvedi, "Flexible and Dynamic Power Allocation in Broadband Multi-Beam Satellites," IEEE Communications Letters, vol. 17, no. 9, pp. 1722-1725, Sep. 2013.

[11] A. Destounis and A. D. Panagopoulos, "Dynamic Power Allocation for Broadband Multi-Beam Satellite Communication Networks," IEEE Communications Letters, vol. 15, no. 4, pp. 380-382, April 2011.

[12] A. I. Aravanis, B. Shankar M. R., P. Arapoglou, G. Danoy, P. G. Cottis, and B. Ottersten, "Power Allocation in Multibeam Satellite Systems: A Two-Stage Multi-Objective Optimization," IEEE Transactions on Wireless Communications, vol. 14, no. 6, pp. 3171-3182, June 2015.

[13] S. Kisseleff, B. Shankar, D. Spano, and J.-D. Gayrard, “A New Optimization Tool for Mega-Constellation Design and its Application to Trunking Systems," in International Communications Satellite Systems Conference (ICSSC), Nov. 2019.

[14] J. Lei and M. A. Vázquez-Castro, "Joint Power and Carrier Allocation for the Multibeam Satellite Downlink with Individual SINR Constraints," in Proc. of IEEE International Conference on Communications, May 2010, pp. 1-5.

[15] _ - "Multibeam satellite frequency/time Duality study and capacity optimization," Journal of Communications and Networks, vol. 13, no. 5 , pp. 472-480, Oct 2011.

[16] X. Alberti, J. M. Cebrian, A. Del Bianco, Z. Katona, J. Lei, M. A. Vazquez-Castro, A. Zanus, L. Gilbert, and N. Alagha, "System capacity optimization in time and frequency for multibeam multi-Media Satellite Systems," in Proc. of Advanced Satellite Multimedia Systems Conference and Signal Processing for Space Communications Workshop, Sep. 2010, pp. 226-233.

[17] A. Paris, I. Del Portillo, B. Cameron, and E. Crawley, "A Genetic Algorithm for Joint Power and Bandwidth Allocation in Multibeam Satellite Systems," in 2019 IEEE Aerospace Conference, March 2019, pp. $1-15$.

[18] G. Cocco, T. de Cola, M. Angelone, Z. Katona, and S. Erl, "Radio Resource Management Optimization of Flexible Satellite Payloads for DVB-S2 Systems," IEEE Transactions on Broadcasting, vol. 64, no. 2, pp. 266-280, Jun. 2018.

[19] K. Roth, H. Pirzadeh, A. L. Swindlehurst, and J. A. Nossek, "A Comparison of Hybrid Beamforming and Digital Beamforming With Low-Resolution ADCs for Multiple Users and Imperfect CSI," IEEE Journal of Selected Topics in Signal Processing, vol. 12, no. 3, pp 484-498, June 2018.

[20] C. N. Efrem and A. D. Panagopoulos, "Dynamic Energy-Efficient Power Allocation in Multibeam Satellite Systems," IEEE Wireless Communications Letters, vol. 9, no. 2, pp. 228-231, 2020.

[21] S. Boyd and L. Vandenberghe, Convex Optimization. Cambridge, UK: Cambridge Univ. Press, 2004.

[22] X. Shen, S. Diamond, Y. Gu, and S. Boyd, "Disciplined Convex-Concave Programming," Apr. 2016, arXiv:1604.02639.

[23] T. Wang and L. Vandendorpe, "Successive convex approximation based methods for dynamic spectrum management," in Proc. of IEEE International Conference on Communications, June 2012, pp. 4061-4065.

[24] K. I. Pedersen, F. Frederiksen, C. Rosa, H. Nguyen, L. G. U. Garcia, and Y. Wang, "Carrier aggregation for LTE-advanced: functionality and performance aspects," IEEE Communications Magazine, vol. 49, no. 6, pp. 89-95, June 2011. 